\title{
Pioglitazone affects the OPG/RANKL/RANK system and increase osteoclastogenesis
}

\author{
FEI XU, YONGHUI DONG, XIN HUANG, PENG CHEN, FENGJING GUO, \\ ANMIN CHEN and SHILONG HUANG \\ Department of Orthopedics, Tongji Hospital Affiliated to Tongji Medical College, \\ Huazhong University of Science and Technology, \\ Wuhan, Hubei 430030, P.R. China
}

Received June 11, 2015; Accepted April 7, 2016

DOI: $10.3892 / \mathrm{mmr} .2016 .5515$

\begin{abstract}
Thiazolidinediones are traditional anti-diabetic therapeutic agents that have been associated with bone loss and increased fracture risk. However, the underlying mechanisms of this side effect require further elucidation. The present study aimed to investigate the effect of pioglitazone (PIO), a thiazolidinedione, on osteoblastogenesis, osteoclastogenesis and the osteoprotegerin $(\mathrm{OPG}) /$ receptor activator of nuclear factor $-\kappa \mathrm{B}$ ligand (RANKL) / RANK system. The MC3T3-E1 murine pre-osteoblastic cell line was treated with $\mathrm{PIO}$ and processed for reverse transcription-quantitative polymerase chain reaction (RT-qPCR) analysis of OPG, RANKL, peroxisome proliferator-activated receptor $\gamma$ (PPAR $\gamma)$, Runt-related transcription factor 2 (RUNX2), alkaline phosphatase (ALP) and osteocalcin (OCN), and western blotting analysis of OPG and RANKL. The culture medium was collected for ELISA analysis of OPG and RANKL. Murine bone marrow monocytes (BMMCs) were treated with PIO in the presence of RANKL and macrophage-colony stimulating factor and subjected to tartrate-resistant acid phosphatase (TRAP) staining and activity measurement, and RT-qPCR analysis of cathepsin K, TRAP and RANK. Co-culture of MC3T3-E1 and BMMCs was performed in the presence of PIO, and TRAP staining was also conducted. PIO inhibited the osteoblastic differentiation of MC3T3-E1 cells, and promoted the osteoclastic differentiation of BMMCs with or without co-culturing with MC3T3-E1 cells. ELISA analysis indicated increased RANKL and decreased OPG expression levels in the medium of MC3T3-E1 cells treated with PIO.
\end{abstract}

Correspondence to: Professor Shilong Huang, Department of Orthopedics, Tongji Hospital Affiliated to Tongji Medical College, Huazhong University of Science and Technology, 1095 Jiefang Avenue, Wuhan, Hubei 430030, P.R. China

E-mail: doctorhsl@gmail.com

Key words: thiazolidinedione, receptor activator of nuclear factor- $\mathrm{\kappa} \mathrm{B}$ ligand, osteoprotegerin, osteoclast, osteoblast
PIO upregulated expression of RANKL and PPAR $\gamma$ and downregulated expression of OPG, RUNX2, ALP and OCN in MC3T3-E1 cells, while expression levels of RANK in $\mathrm{BMMCs}$ remained unchanged. These results suggest that PIO suppresses osteoblastogenesis and enhances osteoclastogenesis. In addition, PIO may also promote osteoclastogenesis by affecting the OPG-RANKL-RANK system.

\section{Introduction}

Thiazolidinediones (TZDs) are therapeutic agents commonly used to treat patients with type 2 diabetes, they have been demonstrated to affect bone metabolism (1). It has been reported that long-term usage of TZDs in diabetic patients induced bone loss and a higher risk of fracture $(2,3)$.

Bone homeostasis depends on the balance between bone formation by osteoblasts and bone resorption by osteoclasts (4). Bone loss and osteoporosis occur when bone resorption exceeds bone formation, and osteopetrosis may occur when bone formation predominates (5). The molecular osteoprotegerin $(\mathrm{OPG}) /$ receptor activator of nuclear factor- $\kappa \mathrm{B}$ ligand (RANKL) / RANK axis is important in the regulation of homeostasis (6). OPG and RANKL are secreted by osteoblasts, RANKL mediates osteoclastogenesis by binding RANK expressed by osteoclasts, whereas OPG acts as a decoy receptor of RANKL to prevent osteoclastogenesis (6). Thus, osteoclastogenesis depends on the ratio of OPG to RANKL secreted by osteoblasts $(6,7)$.

TZDs act via peroxisome proliferator-activated receptor $\gamma$ $(\operatorname{PPAR} \gamma)$, which is a member of the nuclear receptor superfamily of transcription factors (8), and the predominant factor involved in adipose metabolism. Activation of PPAR $\gamma$ results in adipogenic differentiation in various kinds of progenitor cells $(9,10)$. A number of studies have demonstrated that TZDs inhibit osteoblastogenesis directly, but its effect on osteoclastogenesis remains to be determined. Few studies have investigated the effect of TZDs on the OPG/RANKL/RANK system and the paracrine regulation of osteoclastogenesis.

The present study investigated the direct effect of pioglitazone (PIO), a TZD, on osteoblastogenesis and osteoclastogenesis, and the paracrine mechanisms by which 
PIO affects osteoclastogenesis were also investigated by performing a co-culture system of a pre-osteoblastic cell line with bone marrow mononuclear cells.

\section{Materials and methods}

Reagents. Recombinant murine macrophage colony-stimulating factor (M-CSF) and RANKL were purchased from R\&D Systems, Inc. (Minneapolis, MN, USA). The ALP Staining kit and TRAP Staining kit were obtained from Sigma-Aldrich (St. Louis, MO, USA). Anti-RANKL (\#4816; rabbit polyclonal; 1:1,000) antibody was obtained from Cell Signaling Technology, Inc. (Danvers, MA, USA) and the anti-OPG (BA1475-1; rabbit polyclonal; 1:100-400) antibody, OPG and RANKL ELISA kits were obtained from Wuhan Boster Biological Technology, Co., Ltd. (Wuhan, China). PIO was obtained from Santa Cruz Biotechnology, Inc. (Dallas, TX, USA).

Cell culture. The MC3T3-E1 murine pre-osteoblastic cell line was purchased from the American Type Culture Collection (Manassas, VA, USA). Cells were cultured in $\alpha$-modified essential medium ( $\alpha$-MEM; Hyclone; GE Healthcare Life Sciences, Logan, UT, USA) supplemented with $100 \mathrm{U} / \mathrm{ml}$ penicillin, $100 \mu \mathrm{g} / \mathrm{ml}$ streptomycin and $10 \%$ fetal calf serum (FCS; Gibco; Thermo Fisher Scientific, Inc., Waltham, MA, USA) at $37^{\circ} \mathrm{C}$ in a humidified atmosphere of $5 \% \mathrm{CO}_{2}$. The media was changed every 3 days.

Isolation of monocytes. Bone marrow cells were isolated from the femora of C57BL/6 mice (male; age, 6-8 weeks; weight, 18-22 g; Beijing HFK Bioscience Co., Ltd., Beijing, China). The mice were housed separately in a temperature- and humidity-controlled $\left(20-26^{\circ} \mathrm{C}\right.$ and $40-70 \%$, respectively) environment, with a 12/12 $\mathrm{h} \mathrm{light/dark} \mathrm{cycle} \mathrm{and} \mathrm{free} \mathrm{access} \mathrm{to}$ food and water. The mice were sacrificed by $\mathrm{CO}_{2}$ inhalation and cervical dislocation. The cells were cultured in $\alpha$-MEM supplemented with $100 \mathrm{U} / \mathrm{ml}$ penicillin, $100 \mu \mathrm{g} / \mathrm{ml}$ streptomycin and $15 \% \mathrm{FCS}$ at $37^{\circ} \mathrm{C}$ in a humidified atmosphere of $5 \% \mathrm{CO}_{2}$. After $24 \mathrm{~h}$ of culture, the non-adherent BMMCs were collected. All procedures were approved by the Animal Care and Use Committee, Tongji Medical College, Huazhong University of Science and Technology (Wuhan, China).

Osteoblast differentiation. For differentiation studies, MC3T3-E1 cells were cultured in 6-well plates (Corning Incorporated, Corning, NY, USA) at a density of $5 \times 10^{6}$ cells/well. After $24 \mathrm{~h}$, the culture medium was replaced with conditioned medium [ $\alpha$-MEM supplemented with $10 \%$ FCS, $10 \mathrm{mM}$ $\beta$-glycerophosphate (Sigma-Aldrich), $50 \mu \mathrm{g} / \mathrm{ml} \mathrm{L}$-ascorbic acid (Sigma-Aldrich) and $100 \mathrm{nM}$ dexamethasone (Wuhan Boster Biological Technology, Co., Ltd.)] with dimethyl sulfoxide (DMSO; as control; Santa Cruz Biotechnology, Inc.), PIO $0.1 \mu \mathrm{M}$ or PIO $1 \mu \mathrm{M}$ for 14 days. The media were changed every 3 days. For the $24 \mathrm{~h}$ prior to harvesting, the medium of each well was changed to $2 \mathrm{ml}$ conditioned medium without FCS, and the medium was collected for ELISA analysis. The harvested cells were used to conduct ALP staining and activity measurement, reverse transcription-quantitative polymerase chain reaction (RT-qPCR) and western blotting.
Osteoclast differentiation. BMMCs were cultured in 96-well plates (Corning Incorporated) at a density of 5,000 cells/well in conditioned medium containing $50 \mathrm{ng} / \mathrm{ml} \mathrm{M-CSF}$ and $50 \mathrm{ng} / \mathrm{ml}$ RANKL and were treated with DMSO, $0.1 \mu \mathrm{M}$ PIO or $1 \mu \mathrm{M}$ PIO. After 4 days, the cells were harvested and TRAP staining and activity measurement, and western blotting were conducted.

$O P G$ and RANKL ELISA. OPG and RANKL levels in the medium of osteoblast differentiation were analyzed using a commercially available ELISA kit according to the manufacturer's protocols. Each sample was assessed 3 times.

ALP activity and TRAP activity measurement. For measurement of ALP activity, the harvested cells were washed with phosphate-buffered saline (PBS; Gibco; Thermo Fisher Scientific, Inc.) twice prior to the addition of lysis buffer [1.5 M Tris- $\mathrm{HCl}$ ( $\mathrm{pH}$ 9.2), 0.1 $\mathrm{M} \mathrm{ZnCl}_{2}, 0.5 \mathrm{M} \mathrm{MgCl}_{2}-6 \mathrm{H}_{2} \mathrm{O}$, and Triton X-100; Wuhan Boster Biological Technology, Co., Ltd.], and the cells in each well were sonicated for $10 \mathrm{sec}$. The sonicated samples were added to a substrate solution containing 4-nitrophenyl phosphate (Sigma-Aldrich), $1.5 \mathrm{M}$ alkaline buffer solution (Sigma-Aldrich) and $\mathrm{H}_{2} \mathrm{O}$ and incubated for $20 \mathrm{~min}$ at $37^{\circ} \mathrm{C}$. Subsequently, $2 \mathrm{~N} \mathrm{NaOH}$ was added to the samples to stop the reaction. Absorbance of the samples was measured at a wavelength of $405 \mathrm{~nm}$ using a microplate reader (Multiskan ${ }^{\mathrm{TM}}$ FC; Thermo Fisher Scientific, Inc.).

For measurement of TRAP activity, the harvested cells were fixed with $10 \%$ formalin (Sigma-Aldrich) for $10 \mathrm{~min}$ and $95 \%$ ethanol for $1 \mathrm{~min}$, and then $100 \mu \mathrm{l}$ of citrate buffer (50 mM; pH 4.6; Sigma-Aldrich) containing $10 \mathrm{mM}$ sodium tartrate (Sigma-Aldrich) and $5 \mathrm{mM}$ p-nitrophenylphosphate (Sigma-Aldrich) was added to the wells containing fixed cells in the plates. Following incubation for $1 \mathrm{~h}$, enzyme reaction mixtures in the wells were transferred to new plates containing an equal volume of $0.1 \mathrm{~N} \mathrm{NaOH}$. Absorbance was measured at a wavelength of $405 \mathrm{~nm}$ using a Multiskan ${ }^{\mathrm{TM}}$ FC. Each experiment was performed in triplicate.

Co-culture of BMMCs with MC3T3-E1 cells. Using a 6-well Transwell plate (Corning Incorporated), MC3T3-E1 cells were cultured in the lower layer at a density of $5 \times 10^{6}$ cells/well. After $24 \mathrm{~h}$, the $\alpha$-MEM medium was changed to $\alpha$-MEM medium containing DMSO, $0.1 \mu \mathrm{M}$ PIO or $1 \mu \mathrm{M}$ PIO, and BMMCs were cultured in the upper layer of the Transwell plate at the density of 5,000 cells/well. The media was changed every 3 days. After 7 days, the cells in the upper layer were harvested to conduct TRAP staining.

$R N A$ isolation and RT-qPCR analysis. Total RNA was isolated using TRIzol (Invitrogen; Thermo Fisher Scientific, Inc.). DNase I, RNase-free (\#EN0521) and the RevertAid First Strand cDNA Synthesis kit (all from Thermo Fisher Scientific, Inc.) were used for reverse transcription. The reverse trancription reaction was conducted with total RNA, Oligo (dT)18 primers and nuclease-free water made up to a total volume of $12 \mu \mathrm{l}$. This mixture was then incubated at $65^{\circ} \mathrm{C}$ for $5 \mathrm{~min}$, chilled on ice, spun down and placed back on ice. The $5 \mathrm{X}$ reaction buffer $(4 \mu \mathrm{l})$, RiboLock RNase Inhibitor $(1 \mu \mathrm{l}), 10 \mathrm{mM}$ dNTP Mix $(2 \mu \mathrm{l})$, RevertAid M-MuLV RT (200 U/ $\mu \mathrm{l} ; 1 \mu \mathrm{l})$ were then added to a total volume of $20 \mu 1$, the mixture was mixed gently and 
Table I. List of primers.

\begin{tabular}{|c|c|c|c|}
\hline Gene & Primer & Product size & Accession no. \\
\hline $18 \mathrm{~S}$ & $\begin{array}{l}\text { F: TTCGAACGTCTGCCCTATCAA } \\
\text { R: ATGGTAGGCACGGGGACTA }\end{array}$ & 50 & M35283.1 \\
\hline $\operatorname{PPAR} \gamma$ & $\begin{array}{l}\text { F: GGAAGACCACTCGCATTCCTT } \\
\text { R: GTAATCAGCAACCATTGGGTCA }\end{array}$ & 121 & NM_001127330 \\
\hline RUNX2 & $\begin{array}{l}\text { F: GACTGTGGTTACCGTCATGGC } \\
\text { R: ACTTGGTTTTTCATAACAGCGGA }\end{array}$ & 84 & NM_001146038 \\
\hline ALP & $\begin{array}{l}\text { F: GCCTTACCAACTCTTTTGTGCC } \\
\text { R: GCTTGCTGTCGCCAGTAAC }\end{array}$ & 61 & NM_007431 \\
\hline $\mathrm{OCN}$ & $\begin{array}{l}\text { F: CTGACCTCACAGATCCCAAGC } \\
\text { R: TGGTCTGATAGCTCGTCACAAG }\end{array}$ & 187 & NM_031368 \\
\hline RANK & $\begin{array}{l}\text { F: CCAGGAGAGGCATTATGAGCA } \\
\text { R: ACTGTCGGAGGTAGGAGTGC }\end{array}$ & 94 & AF019046.1 \\
\hline Cathepsin K & $\begin{array}{l}\text { F: GAAGAAGACTCACCAGAAGCAG } \\
\text { R: CTGTATTCCCCGTTGTGTAGC }\end{array}$ & 136 & NM_007802 \\
\hline TRAP & $\begin{array}{l}\text { F: CACTCCCACCCTGAGATTTGT } \\
\text { R: CATCGTCTGCACGGTTCTG }\end{array}$ & 118 & NM_001102405 \\
\hline
\end{tabular}

Primers are listed in the 5'-3' direction, and product lengths are presented with the units 'base pairs'. PAR $\gamma$, peroxisome proliferator-activated receptor $\gamma$; RUNX2, Runt-related transcription factor 2; ALP, alkaline phosphatase; OCN, osteocalcin; RANK, receptor activator of nuclear factor- $\kappa \mathrm{B}$; TRAP, tartrate-resistant acid phosphatase; F, forward; R, reverse.

A

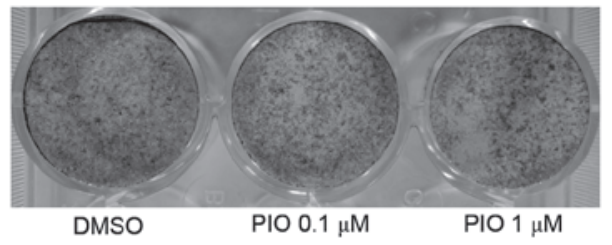

C

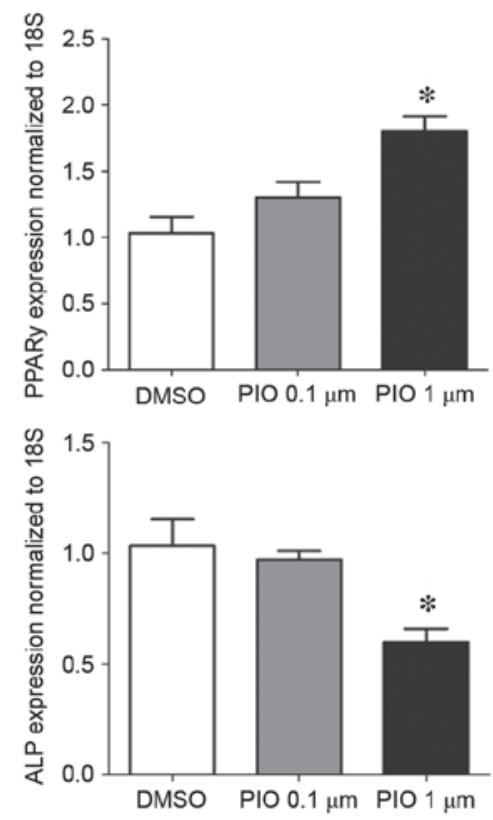

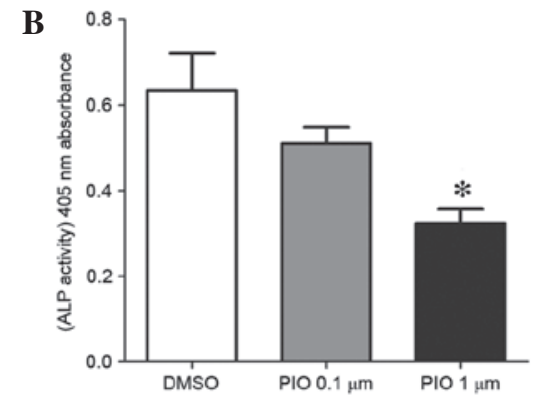
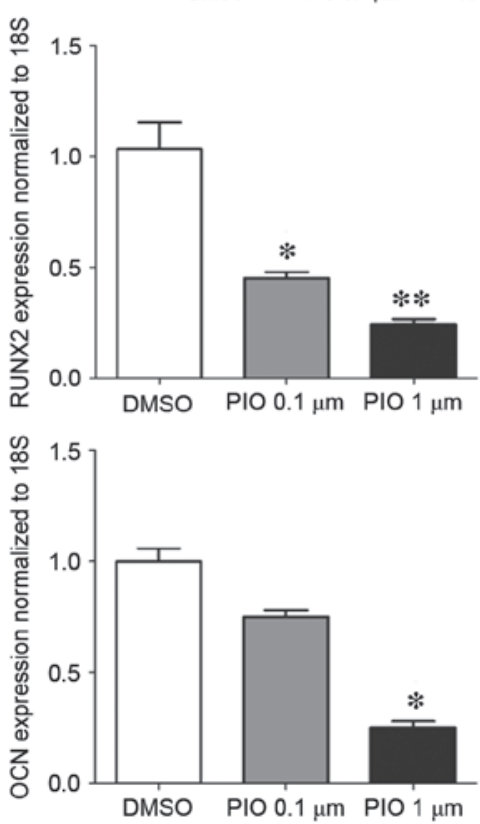

Figure 1. In vitro analysis of the effect of pioglitazone on osteoblasts. (A) ALP staining of osteoblasts at different pioglitazone concentrations. (B) ALP activities of osteoblast, as a quantitative index. (C) The effect of pioglitazone on gene expression in osteoblasts as analyzed by reverse transcription-quantitative polymerase chain reaction. ${ }^{*} \mathrm{P}<0.05$ vs. control; ${ }^{* *} \mathrm{P}<0.01$ vs. control. ALP, alkaline phosphatase; DMSO, dimethyl sulfoxide; PIO, pioglitazone; PPAR $\gamma$, peroxisome proliferator-activated receptor $\gamma$; RUNX2, Runt-related transcription factor 2; OCN, osteocalcin. 
A

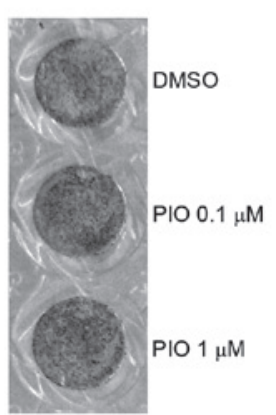

B

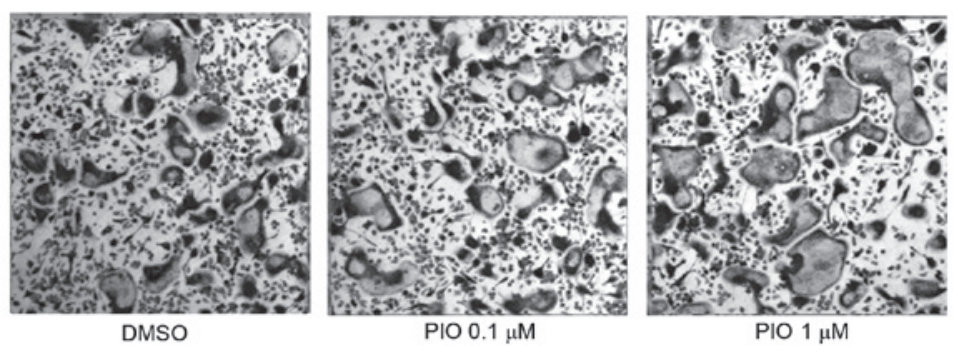

C

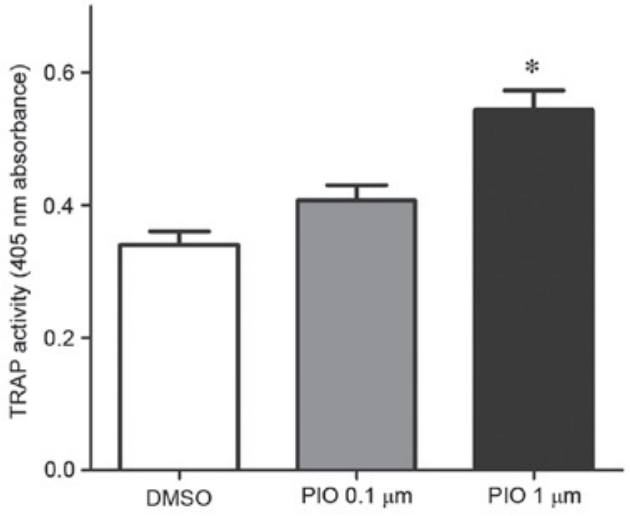

$\mathbf{E}$

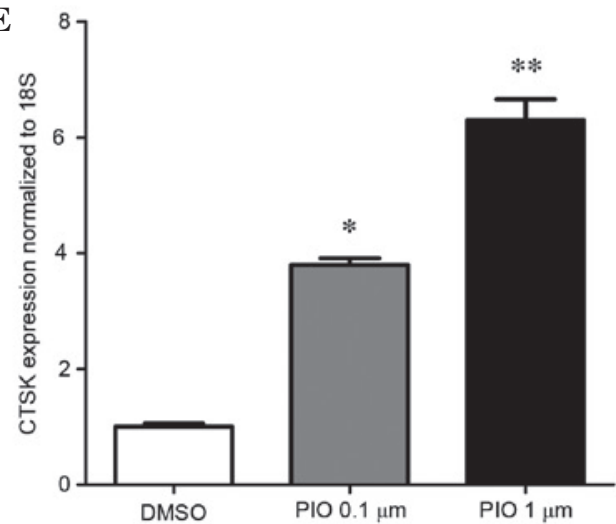

D
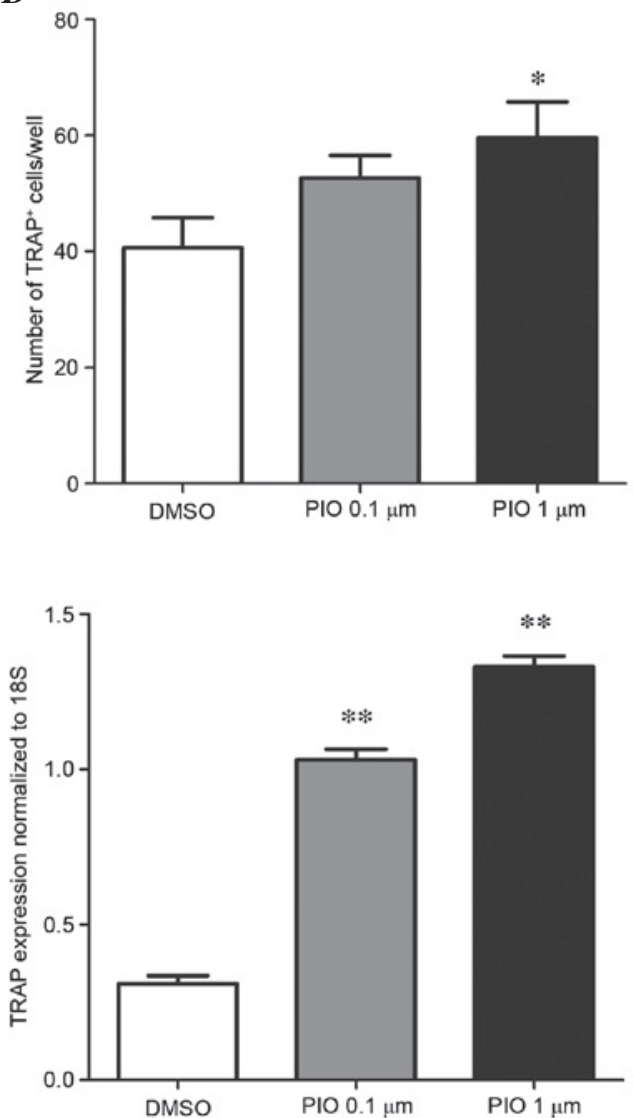

Figure 2. In vitro analysis of pioglitazone effects on osteoclastogenesis. (A and B) Observation of TRAP-positive osteoclasts after 4 days of culture in the presence of macrophage-colony stimulating factor, receptor activator of nuclear factor- $\mathrm{B}$ ligand and different concentrations of PIO. (C) TRAP activity in osteoclasts, as a quantitative index. (D) Counted number of TRAP-positive cells. (E) The effect of PIO on gene expression in osteoblasts was analyzed by reverse transcription-quantitative polymerase chain reaction. ${ }^{*} \mathrm{P}<0.05$ vs. control; ${ }^{* *} \mathrm{P}<0.01$ vs. control. TRAP, tartrate-resistant acid phosphatase; $\mathrm{DMSO}$, dimethyl sulfoxide; PIO, pioglitazone; CTSK, cathepsin K.

centrifuged prior to incubation for $60 \mathrm{~min}$ at $42^{\circ} \mathrm{C}$. For $\mathrm{qPCR}$, Thermo Fisher Scientific, Inc. Maxima SYBR Green qPCR Master Mix (2X) Thermal cycling was conducted, which used a three-step cycling protocol: Pre-treatment at $50^{\circ} \mathrm{C}$ for $2 \mathrm{~min}$, 1 cycle of $95^{\circ} \mathrm{C}$ for $10 \mathrm{~min}$; 1 cycle of denaturation at $95^{\circ} \mathrm{C}$ for $15 \mathrm{sec} ; 40$ cycles of annealing at $60^{\circ} \mathrm{C}$ for $30 \mathrm{sec}$ and extension at $72^{\circ} \mathrm{C}$ for $30 \mathrm{sec}$. The forward and reverse primers were obtained from Invitrogen (Thermo Fisher Scientific, Inc.) and are presented in Table I. Data are presented as the mean \pm standard deviation for at least three independent experiments. Gene expression analysis was performed using RT-qPCR (iCycler iQ5 System; Bio-Rad Laboratories, Inc., Hercules, CA, USA) and normalized to $18 \mathrm{~S}$ RNA.
ALP and TRAP staining. Harvested cells were rinsed three times with PBS and fixed for $15 \mathrm{~min}$ in $4 \%$ paraformaldehyde (Wuhan Boster Biological Technology, Co., Ltd.) at $4^{\circ} \mathrm{C}$. The cells were stained with ALP and TRAP using kits according to the manufacturer's protocols. The staining was analyzed using an OsteoMeasure system (Osteometrics, Inc., Atlanta, GA, USA) connected to a Axioskop microscope (Zeiss, Oberkochen, Germany).

Western blotting. Total cell lysates were obtained by lysing cells in radioimmunoprecipitation assay buffer (Wuhan Boster Biological Technology, Co., Ltd.) containing $50 \mathrm{mM}$ Tris- $\mathrm{HCl}, 150 \mathrm{mM} \mathrm{NaCl}, 1 \% \mathrm{NP}-40,0.1 \%$ SDS, $0.5 \%$ sodium 
Table II. The OPG and RANKL levels in osteoblast culture medium (detected by ELISA).

\begin{tabular}{lccr}
\hline Gene & DMSO $(\mathrm{n}=3)$ & PIO $0.1 \mu \mathrm{M}(\mathrm{n}=3)$ & PIO $1 \mu \mathrm{M}(\mathrm{n}=3)$ \\
\hline OPG $(\mathrm{pg} / \mathrm{ml})$ & $476.5 \pm 26.8$ & $431.6 \pm 26.18$ & $326.12 \pm 34.4^{\mathrm{a}}$ \\
RANKL $(\mathrm{pg} / \mathrm{ml})$ & $792.5 \pm 43.1$ & $815.5 \pm 33.2$ & $1265.1 \pm 79.2^{\mathrm{b}}$
\end{tabular}

${ }^{\mathrm{a}} \mathrm{P}<0.05$; ${ }^{\mathrm{b}} \mathrm{P}<0.01$. OPG, osteoprotegerin; RANKL, receptor activator of nuclear factor- $\kappa \mathrm{B}$ ligand; DMSO, dimethyl sulfoxide; PIO, pioglitazone.

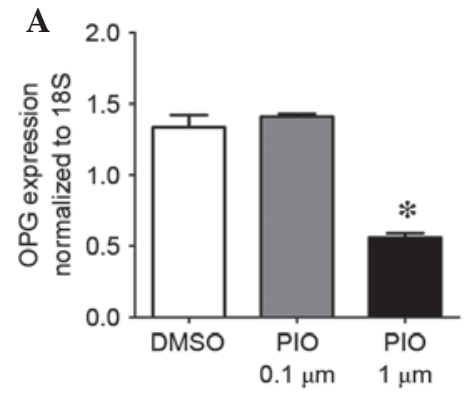

C

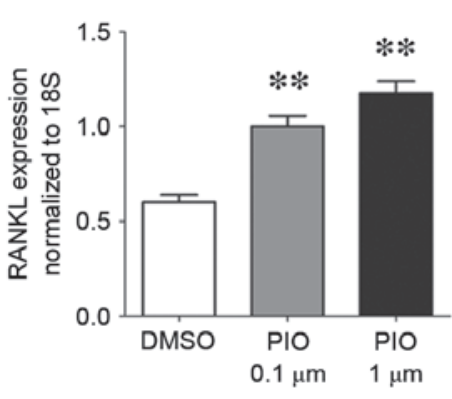

D
B

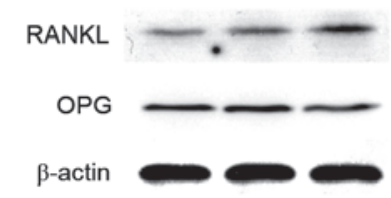

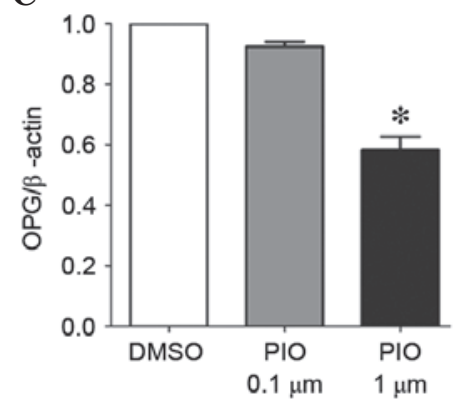

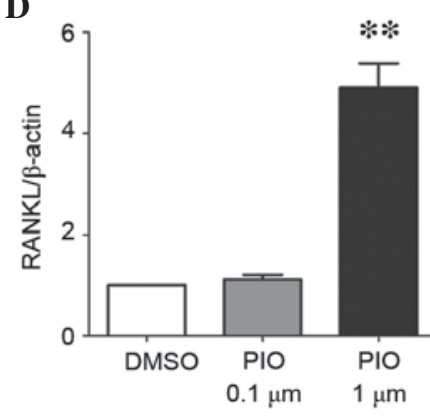

Figure 3. Effects of PIO on the OPG/RANKL/RANK system on osteoblasts and osteoclasts. (A) Gene expression in osteoblasts and osteoclasts stimulated by PIO. (B) The effect of PIO on protein expression, OPG and RANKL, in osteoblast and analyzed by western blot. (C) The OPG and RANKL expression in osteoblast quantified. (D) The OPG/RANKL ratio in osteoblasts was decreased by PIO. " $\mathrm{P}<0.05$ vs. control; ${ }^{* *} \mathrm{P}<0.01$ vs. control. PIO, pioglitazone; OPG, osteoprotegerin; RANKL, receptor activator of nuclear factor- $\kappa \mathrm{B}$ ligand; DMSO, dimethyl sulfoxide.

deoxycholate, $2 \mathrm{mM}$ sodium fluoride, $1 \mathrm{mM}$ EDTA, $1 \mathrm{mM}$ EGTA and a protease inhibitor cocktail. Protein concentration was determined using a Pierce BCA protein assay kit (Thermo Fisher Scientific, Inc.). The proteins $(20 \mu \mathrm{g})$ were separated by $10 \%$ sodium dodecyl sulfate-polyacrylamide gel electrophoresis, transferred to nitrocellulose membranes, blocked with bovine serum albumin (Wuhan Boster Biological Technology, Co., Ltd.) in Tris-buffered saline with $0.1 \%$ Tween-20 (TBST) for $1 \mathrm{~h}$ at room temperature, and incubated with primary antibodies (anti-OPG and anti-RANKL) overnight at $4^{\circ} \mathrm{C}$. The membrane was washed three times with TBST and incubated with the horseradish peroxidase-conjugated secondary antibodies (BA1039; goat anti-rabbit monoclonal; 1:1,000; Wuhan Boster Biological Technology, Co., Ltd.) for $1 \mathrm{~h}$ at room temperature, then washed three further times with TBST. Protein detection was conducted with a Pierce enhanced chemiluminescence detection system (Thermo Fisher Scientific, Inc.). Densitometry was conducted using Quantity One software (Bio-Rad Laboratories, Inc.).

Statistical analysis. All data were presented as the mean \pm standard deviation. Statistical analysis was performed using one-way analysis of variance with SPSS software, version 12.0 (SPSS, Inc., Chicago, IL, USA). P $<0.05$ was considered to indicate a statistically significant difference.

\section{Results}

PIO inhibits the differentiation of MC3T3-E1 cells into osteoblasts. To investigate the effect of PIO on osteoblast differentiation, MC3T3-E1 cells were cultured in osteoblastic differentiation media with or without PIO. ALP staining and ALP activity served as biomarkers of osteoblastic differentiation (Fig. 1). As presented in Fig. 1A and B, $1 \mu \mathrm{M}$ PIO significantly reduced the number of ALP positive osteoblasts and ALP activity when compared with the control group $(\mathrm{P}<0.05)$. In addition, expression levels of osteoblastic genes, Runt-related transcription factor 2 (RUNX2) $(\mathrm{P}<0.01)$, ALP $(\mathrm{P}<0.05)$ and osteocalcin $(\mathrm{OCN} ; \mathrm{P}<0.05)$ were significantly decreased following treatment with $1 \mu \mathrm{M}$ PIO (Fig. 1C). By contrast, PPAR $\gamma$ was significantly increased $(\mathrm{P}<0.05$; Fig. 1C). No significant alterations were observed following $0.1 \mu \mathrm{M}$ PIO treatment, apart from in RUNX2 expression. 
A

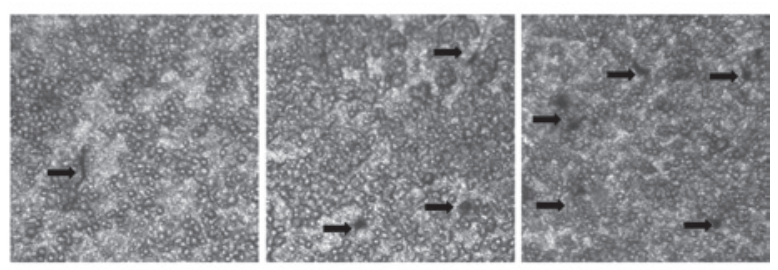

B

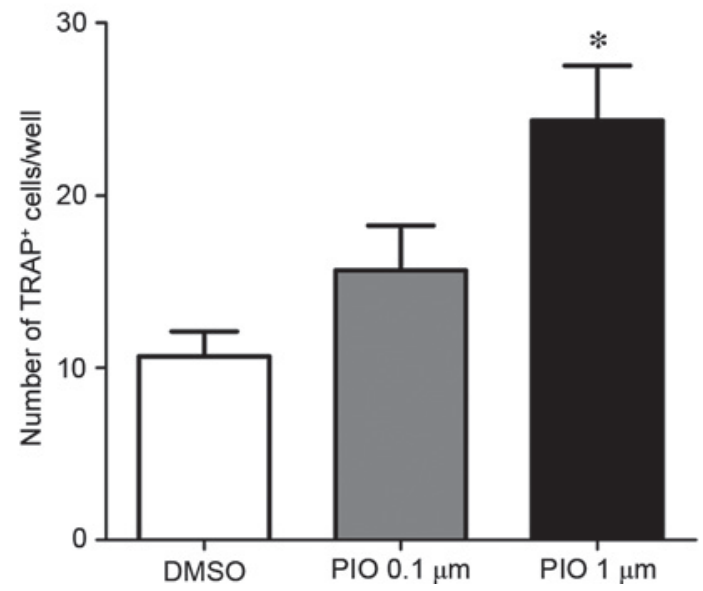

Figure 4. In the co-culture system, PIO was observed to increase osteoclastogenesis of monocytes by osteblasts. (A) Red arrows indicate TRAP-positive cells. PIO and molecules secreted by the osteoblasts increased the TRAP-positive cells compared with the control group.(B) The TRAP activity was detected in the upper layer of the co-culture system, the result demonstrates that the TRAP activity in the PIO groups was increased compared with the control group $\left({ }^{*} \mathrm{P}<0.05\right)$. PIO, pioglitazone; TRAP, tartrate-resistant acid phosphatase.

PIO promotes the differentiation of BMMCs into osteoclasts. The effect of pioglitazone on osteoclast differentiation was investigated in BMMCs by culturing the cells in the presence of RANKL $(50 \mathrm{ng} / \mathrm{ml})$ and M-CSF $(50 \mathrm{ng} / \mathrm{ml})$ with or without PIO, and detecting the number of TRAP-positive cells and TRAP activity, which indicate the degree of osteoclast differentiation (Fig. 2). As presented in Fig. 2A, RANKL-mediated osteoclast differentiation was promoted by $1 \mu \mathrm{M}$ PIO. The number of TRAP-positive cells (Fig. 2C and D) and TRAP activity (Fig. 2B) were significantly increased following treatment with $1 \mu \mathrm{M}$ PIO. In addition, the gene expression levels of TRAP and cathepsin $\mathrm{K}$, which indicate the function of osteoclasts, were significantly increased $(\mathrm{P}<0.05$; Fig. $2 \mathrm{E})$. Treatment with $0.1 \mu \mathrm{M}$ PIO did not significantly affect the osteoclast differentiation of BMMCs.

PIO decreases the OPG/RANKL ratio in osteoblasts. The MC3T3-E1 cells were treated with PIO or DMSO for 7 days, and the mRNA and protein expression levels of OPG and RANKL were analyzed using RT-qPCR and western blotting, respectively. As presented in Fig. 3, the $1 \mu \mathrm{M}$ PIO group exhibited a significant decrease of the ratio of OPG/RANKL mRNA expression levels $(\mathrm{P}<0.05)$ due to decreased OPG and increased RANKL expression levels compared with the control group. Furthermore, the protein expression levels of OPG, RANKL and ratio of OPG to RANKL demonstrated the same trend of change as observed for the mRNA expression levels. The cells treated with $0.1 \mu \mathrm{M}$ PIO demonstrated no significant difference compared with that of the control group.

PIO promotes osteoclastogenesis by modulating the osteoblast and osteoclast cross-talk. The OPG and RANKL expression levels in the osteoblast culture medium of MC3T3-E1 cells were detected by ELISA. Following PIO treatment, OPG expression levels decreased and RANKL expression levels increased, compared with DMSO-treated controls (Table II). Few TRAP positive cells were observed in BMMCs co-cultured with MC3T3-E1 cells without PIO treatment after 7 days (Fig. 4A). Following addition of PIO, an increased number of TRAP positive cells were observed (Fig. 4). Furthermore, the number of TRAP-positive cells among BMMCs in the PIO-treated co-culture system was significantly increased compared with BMMCs untreated with PIO ( $\mathrm{P}<0.05$; Fig. 4B). Notably, in these experiments, $0.1 \mu \mathrm{M}$ PIO demonstrated the same effects as $1 \mu \mathrm{M}$ pioglitazone, however, the difference was not statistically significant (Fig. 4A and B).

\section{Discussion}

Pioglitazone inhibited osteoblast differentiation and promoted osteoclast formation. As one of thiazolidinediones that are commonly prescribed for patients with diabetes, PIO is a PPAR $\gamma$ agonist, which may result in bone loss and an increased fracture rate in diabetic patients (2). The present study demonstrated that PIO inhibited osteoblast differentiation and promoted osteoclast formation directly, and enhanced osteoclastogenesis via the OPG/RANKL/RANK system, which is involved in paracrine regulation of osteoclastogenesis.

PIO negatively regulates osteoblast differentiation. Consistently with previous studies, the present study demonstrated that PIO inhibited osteoblastogenesis. Osteoblasts and adipocytes are commonly derived from mesenchymal stem cells (MSCs), and their differentiation is reliant on different stimulating signals. Activation of core binding factor- $\alpha 1 /$ RUNX 2 results in osteoblast formation from MSCs, however, activation of PPAR $\gamma$ results in adipocyte formation $(11,12)$. Competition exists between the differentiation of the two cell types, previous studies have shown that TDZs promote adipocytogenesis at expense of osteoblastogenesis (13-16). Furthermore, silencing PPAR $\gamma$ using synthetic small interfering RNA inhibited adipocyte differentiation and induced osteoblastic differentiation (17). However, the underlying mechanisms remain to be elucidated. In the present study, PIO was observed to upregulate PPAR $\gamma$ and downregulate RUNX2 in parallel with inhibition of osteoblastogenesis. Results from the current study support the findings of Jeon et al (18) that activation of PPAR $\gamma$ interfered with the transactivation ability of RUNX2 and suppressed its expression, thus resulting in the inhibition of OCN (an osteoblast-specific protein) expression. Furthermore, apoptosis of osteoblasts has been proven to be accelerated by TZDs. By contrast, Bruedigam et al (19) demonstrated that rosiglitazone promoted osteoblastogenesis and produced a large quantity of reactive oxygen species and increased apoptosis, which resulted in attenuation of osteoblastogenesis. 
PIO increases osteoclastogenesis. Osteoclasts are derived from bone marrow hematopoietic stem cells, which may be stimulated to differentiate by RANKL signaling and PPAR $\gamma$ activation (20). TZDs have been reported to affect osteoclast differentiation, however, results from various previous studies are conflicting. Chan et al (21) demonstrated that ciglitazone, a TZD, suppressed multinucleated osteoclast formation in a dose-dependent manner. Furthermore, Cho et al (15) demonstrated that rosiglitazone attenuated osteoclast formation and bone resorption by preventing RANK and enhancing PPAR $\gamma 2$ expression in osteoclasts. By contrast, Wan et al (22) demonstrated that activation of PPAR $\gamma$ stimulated osteoclastogenesis and bone resorption, and deletion of PPAR $\gamma$ prevented osteoclast formation and resulted in osteopetrosis. Wu et al (23) also demonstrated that rosiglitazone markedly increased the differentiation of mice bone marrow cells into osteoclasts. In the present study, administration of exogenous RANKL and M-CSF, which are required for osteoclast differentiation, demonstrated that PIO exerts a direct effect on promoting differentiation of BMMCs into osteoclasts. Furthermore, contrary to results from Cho et al (15), the RANK expression levels in the cells remained unchanged, which indicated that this effect of PIO may involve PPAR $\gamma$ but not the RANKL-RANK response. Although c-Fos induction, TNF receptor-associated factor 6 and downstream extracellular signal-regulated kinase signaling, PPAR $\gamma$ coactivator $1 \beta$ and estrogen-related receptor $\alpha$ have been reported to be associated with enhancement of PPAR $\gamma$-mediated osteoclastogenesis $(22,24)$, the underlying mechanisms remain to be elucidated.

PIO decreases the OPG/RANKL ratio in osteoblasts. The molecular OPG/RANKL/RANK axis participates in regulating bone metabolism, provides paracrine regulation for osteoclastogenesis (25). RANKL, expressed and secreted by osteoblasts, binds the extracellular RANK domain of pre-osteoclasts, and results in expression of specific genes involved in osteoclast differentiation and bone resorption (26). Furthermore, OPG, secreted by osteoblasts, acts as a soluble receptor antagonist for RANKL, to prevent it binding to RANK and decreases osteoclastogenesis (6). Thus, the OPG/RANKL ratio and the expression of RANK in pre-osteoclasts are key in osteoclastogenesis $(6,7)$. However, studies concerning the effect of TZDs on this paracrine regulation are rare.

In the present study, it was observed that the OPG/RANKL ratio decreased, due to a decreased OPG expression level and an elevated RANKL expression level, in osteoblasts in response to treatment with PIO. Furthermore, the expression levels of RANK remained unchanged following PIO treatment. Thus, the results of the present study suggest, in addition to the direct effect, PIO may positively regulate osteoclastogenesis via influencing the OPG/RANKL/RANK axis in the co-culture system mimicking the in vivo bone marrow microenvironment. Consistent with these findings, Lazarenko et al (27) demonstrated that PPAR $\gamma$ activation in osteoblasts promotes osteoclast differentiation by inducing the expression of RANKL. Previous in vitro and in vivo studies have demonstrated that TZD treatment lowers OPG levels (28-30). Contrary to results in the present study,
Cho et al (15) demonstrated that rosiglitazone inhibited the RANK protein expression in monocytes induced by RANKL. This difference may be attributed to the higher dose of TZD used. In future experiments a larger range of doses of TZDs should be evaluated.

In conclusion, the present study demonstrates that PIO suppresses osteoblastogenesis and enhances osteoclastogenesis directly. It also decreases the OPG/RANKL ratio in osteoblasts, to promote osteoclast formation via a paracrine mechanism.

\section{Acknowledgements}

The present study was supported by the National Natural Sciences Research Program of China (grant no. 81070691).

\section{References}

1. Derosa G: Efficacy and tolerability of pioglitazone in patients with type 2 diabetes mellitus: Comparison with other oral antihyperglycaemic agents. Drugs 70: 1945-1961, 2010.

2. McDonough AK, Rosenthal RS, Cao X and Saag KG: The effect of thiazolidinediones on BMD and osteoporosis. Nat Clin Pract Endocrinol Metab 4: 507-513, 2008.

3. Montagnani A and Gonnelli S: Antidiabetic therapy effects on bone metabolism and fracture risk. Diabetes Obes Metab 15: 784-791, 2013.

4. Raggatt LJ and Partridge NC: Cellular and molecular mechanisms of bone remodeling. J Biol Chem 285: 25103-25108, 2010.

5. Lazner F, Gowen M, Pavasovic D and Kola I: Osteopetrosis and osteoporosis: Two sides of the same coin. Hum Mol Genet 8: 1839-1846, 1999.

6. Khosla S: Minireview: The OPG/RANKL/RANK system. Endocrinology 142: 5050-5055, 2001.

7. Eriksen EF: Cellular mechanisms of bone remodeling. Rev Endocr Metab Disord 11: 219-227, 2010.

8. Lehmann JM, Moore LB, Smith-Oliver TA, Wilkison WO, Willson TM and Kliewer SA: An antidiabetic thiazolidinedione is a high affinity ligand for peroxisome proliferator-activated receptor gamma (PPAR gamma). J Biol Chem 270: 12953-12956, 1995.

9. Tontonoz P, Hu E and Spiegelman BM: Stimulation of adipogenesis in fibroblasts by PPAR gamma 2, a lipid-activated transcription factor. Cell 79: 1147-1156, 1994.

10. MacDougald OA and Lane MD: Transcriptional regulation of gene expression during adipocyte differentiation. Annu Rev Biochem 64: 345-373, 1995.

11. Gimble JM, Robinson CE, Wu X, Kelly KA, Rodriguez BR, Kliewer SA, Lehmann JM and Morris DC: Peroxisome proliferator-activated receptor-gamma activation by thiazolidinediones induces adipogenesis in bone marrow stromal cells. Mol Pharmacol 50: 1087-1094, 1996.

12. Mabilleau G, Chappard D and Baslé MF: Cellular and molecular effects of thiazolidinediones on bone cells: A review. Int J Biochem Mol Biol 2: 240-246, 2011.

13. Wang L, Li L, Gao H and Li Y: Effect of pioglitazone on transdifferentiation of preosteoblasts from rat bone mesenchymal stem cells into adipocytes. J Huazhong Univ Sci Technolog Med Sci 32: 530-533, 2012.

14. Liu L, Aronson J and Lecka-Czernik B: Rosiglitazone disrupts endosteal bone formation during distraction osteogenesis by local adipocytic infiltration. Bone 52: 247-258, 2013.

15. Cho ES, Kim MK, Son YO, Lee KS, Park SM and Lee JC: The effects of rosiglitazone on osteoblastic differentiation, osteoclast formation and bone resorption. Mol Cells 33: 173-181, 2012.

16. Viccica G, Francucci CM and Marcocci C: The role of PPAR $\gamma$ for the osteoblastic differentiation. J Endocrinol Invest 33 (Suppl 7): S9-S12, 2010.

17. Yamashita A, Takada T, Nemoto K, Yamamoto G and Torii R: Transient suppression of PPARgamma directed ES cells into an osteoblastic lineage. FEBS Lett 580: 4121-4125, 2006. 
18. Jeon MJ, Kim JA, Kwon SH, Kim SW, Park KS, Park SW, Kim SY and Shin CS: Activation of peroxisome proliferator-activated receptor-gamma inhibits the Runx2-mediated transcription of osteocalcin in osteoblasts. J Biol Chem 278: 23270-23277, 2003.

19. Bruedigam C, Eijken M, Koedam M, van de Peppel J, Drabek K, Chiba $\mathrm{H}$ and van Leeuwen JP: A new concept underlying stem cell lineage skewing that explains the detrimental effects of thiazolidinediones on bone. Stem Cells 28: 916-927, 2010.

20. Teitelbaum SL and Ross FP: Genetic regulation of osteoclast development and function. Nat Rev Genet 4: 638-649, 2003.

21. Chan BY, Gartland A, Wilson PJ, Buckley KA, Dillon JP, Fraser WD and Gallagher JA: PPAR agonists modulate human osteoclast formation and activity in vitro. Bone 40: 149-159, 2007.

22. Wan Y, Chong LW and Evans RM: PPAR-gamma regulates osteoclastogenesis in mice. Nat Med 13: 1496-1503, 2007.

23. Wu H, Li L, Ma Y, Chen Y, Zhao J, Lu Y and Shen P: Regulation of selective PPAR $\gamma$ modulators in the differentiation of osteoclasts. J Cell Biochem 114: 1969-1977, 2013.

24. Wei W, Wang X, Yang M, Smith LC, Dechow PC, Sonoda J, Evans RM and Wan Y: PGClbeta mediates PPARgamma activation of osteoclastogenesis and rosiglitazone-induced bone loss Cell Metab 11: 503-516, 2010.
25. Hofbauer LC, Khosla S, Dunstan CR, Lacey DL, Boyle WJ and Riggs BL: The roles of osteoprotegerin and osteoprotegerin ligand in the paracrine regulation of bone resorption. $\mathrm{J}$ Bone Miner Res 15: 2-12, 2000

26. Muruganandan S, Roman AA and Sinal CJ: Adipocyte differentiation of bone marrow-derived mesenchymal stem cells: Cross talk with the osteoblastogenic program. Cell Mol Life Sci 66: 236-253, 2009.

27. Lazarenko OP, Rzonca SO, Hogue WR, Swain FL, Suva LJ and Lecka-Czernik B: Rosiglitazone induces decreases in bone mass and strength that are reminiscent of aged bone. Endocrinology 148: 2669-2680, 2007.

28. Park JS, Cho MH, Nam JS, Yoo JS, Ahn CW, Cha BS, Kim KR and Lee HC: Effect of pioglitazone on serum concentrations of osteoprotegerin in patients with type 2 diabetes mellitus. Eur J Endocrinol 164: 69-74, 2011.

29. Sultan A, Avignon A, Galtier F, Piot C, Mariano-Goulart D, Dupuy AM and Cristol JP: Osteoprotegerin, thiazolidinediones treatment and silent myocardial ischemia in type 2 diabetic patients. Diabetes Care 31: 593-595, 2008.

30. Krause U, Harris S, Green A, Ylostalo J, Zeitouni S, Lee N and Gregory CA: Pharmaceutical modulation of canonical Wnt signaling in multipotent stromal cells for improved osteoinductive therapy. Proc Natl Acad Sci USA 107: 4147-4152, 2010. 